\title{
Percepción de los estudiantes en pre-práctica de la Facultad de Educación sobre el rol del profesional en bibliotecología escolar en el proceso de enseñanza y aprendizaje
}

\author{
Perception of Students in Pre-practice from the Faculty of Education on the \\ Role of School Librarians in the Teaching and Learning Process
}

\author{
Noraida Domínguez Flores ${ }^{*}$ \\ Escuela Graduada de Ciencias y Tecnologías de la Información de la \\ Universidad de Puerto Rico. \\ Eileene R. Adames Méndez ${ }^{\star *}$ \\ Escuela Graduada de Ciencias y Tecnologías de la Información de la \\ Universidad de Puerto Rico. \\ Damaris Arocho Molina ${ }^{* * *}$ \\ Escuela Graduada de Ciencias y Tecnologías de Información de la \\ Universidad de Puerto Rico.
}

Recibido: 25 de agosto 2015 Aceptado: 04 de marzo 2016

Corregido: 09 de mayo 2016 Publicado: 24 de junio 2016

Resumen

El presente artículo discute la necesidad de educar a futuros maestros sobre el rol de la biblioteca escolar en los procesos de enseñanza y aprendizaje. Específicamente, la necesidad de que los futuros maestros aprendan a cómo colaborar con los profesionales en bibliotecología escolar en la enseñanza de las competencias de información. Considerando esta necesidad, se desarrolló un proyecto de colaboración que fue integrado en un curso de prepráctica de la Facultad de Educación de la Universidad de Puerto Rico, con la participación de estudiantes del curso de Competencias Informaciones y Tecnológicas de la Escuela Graduada de Ciencias y Tecnologías de la Información del mismo recinto.

Los hallazgos de esta investigación evidenciaron que los futuros maestros carecen de conocimiento en torno a las competencias de información, así como sobre el rol de los profesionales en bibliotecología escolar en los procesos de enseñanza y aprendizaje. Al finalizar el proyecto se evidenció un impacto positivo sobre la percepción de los futuros maestros hacia la biblioteca escolar y las futuras relaciones de colaboración que pueden desarrollarse, una vez iniciada su vida profesional como maestros.

Palabras clave

Bibliotecas escolares, competencias de información, Rol de la Biblioteca Escolar, Rol de Bibliotecarios Escolares, Proyectos de Colaboración.

\section{bibliotecas}


Noraida Domínguez Flores

Eileene R. Adames Méndez
Bibliotecas. Vol 34, N 1, enero-junio, 2016, pp. 1-18. EISSN: 1659-3286

URL: http://www.revistas.una.ac.cr/index.php/bibliotecas/index

\begin{abstract}
This article discusses the need for educating future teachers on the role of the school library in the processes of teaching and learning. Specifically, the need for future teachers to learn how to collaborate with the (ACE) library (ACE) school in the teaching of information skills. Whereas this need, developed a collaborative project that was integrated into a course of pre-practice for the Faculty of education of the University of Puerto Rico, with the participation of students of the course competencies information and technology of the Graduate School of science and information technology from the same venue. The findings of this research showed that future teachers librarians (as) lack of knowledge around the competencies of information, as well as on the role of the (ACE) school in the processes of teaching and learning. At the end of the project showed a positive impact on the perception of future teachers to the school library and future collaborative relationships that can develop after starting his professional career.
\end{abstract}

\title{
Keywords
}

School Libraries, Information Literacy, Role of the School Library, Role of School Librarians, Collaborative Projects.

\section{Introducción}

La biblioteca escolar es el espacio donde converge la necesidad informativa y la satisfacción de esta con el desarrollo de la imaginación, el conocimiento y el pensamiento crítico. Todos estos elementos se entrelazan para lograr que el estudiante no solo sea un aprendiz responsable, sino un colaborador eficaz del aprendizaje continuo en nuestra sociedad.

En las últimas décadas, hemos sido etiquetados como sociedad de la información, debido a la creciente explosión y exposición informática en la que nos vemos sumergidos diariamente gracias al uso del Internet y los medios tecnológicos. La biblioteca escolar no ha sido excluida de esta avalancha tecnológica y, por ello, ha sido indispensable la adaptación de su personal, sus servicios y recursos para mantenerse emergente como principal medio de información en las escuelas.

Según el Manifiesto de la UNESCO (1999), con el fin de instruir eficazmente a sus usuarios, para el bibliotecario escolar será esencial el uso de habilidades de programación y organización de los diferentes recursos de información accesibles en su entorno, pero para garantizar esto será indispensable la capacitación profesional continúa.

Consciente de la necesidad de capacitar eficazmente a los estudiantes y que estos adquieran destrezas informativas que le permitan utilizar la tecnología que les rodea, de forma responsable y así respaldar el proceso de aprendizaje, el Comité de Acreditación y Criterios Profesionales de la Sociedad Internacional para la Tecnología en la Educación (ISTE), creólos Estándares Nacionales de Tecnologías de Información y Comunicación para la Educación (NETS). Estos estándares sirven de guía a los bibliotecarios y maestros para conocer cuáles son las destrezas y habilidades que los estudiantes necesitan aprender para manejar de forma útil las TICs (Tecnologías de Información y Comunicación).

\section{bibliotecas}


Noraida Domínguez Flores

Eileene R. Adames Méndez
Bibliotecas. Vol 34, № 1, enero-junio, 2016, pp. 1-18. EISSN: 1659-3286

URL: http://www.revistas.una.ac.cr/index.php/bibliotecas/index

Según ISTE (2007) los estudiantes deberían saber y ser capaces de aprender efectivamente y vivir productivamente en un mundo cada vez más digital. Para lograr esto, NETS organizó en seis estándares las competencias tecnológicas que cada estudiante debe poseer: creatividad e innovación, comunicación y colaboración, investigación y manejo de la información, pensamiento crítico, solución de problemas y toma de decisiones, ciudadanía digital y operaciones y conceptos en las TIC (EDUTECA, 2008).

Estos estándares son un reflejo de la teoría constructivista en la cual se percibe al estudiante no como un simple receptor del conocimiento, sino como un constructor de éste. En el proceso de aprendizaje según esta teoría, el estudiante utiliza los diferentes estímulos, experiencias y conocimientos previos para la adquisición de los nuevos. En el constructivismo, el estudiante crea, cambia y ajusta sus esquemas de conocimiento, adquiere y vivencia nuevas experiencias, las cuales considera significativas para su realidad. El rol del maestro en este proceso es de facilitador del aprendizaje, guía y mentor del estudiante y ofrece los estímulos necesarios para garantizar pertinencia en la enseñanza de cada aprendiz.

En Puerto Rico, el Departamento de Educación mediante el Programa de Servicio Bibliotecario y de Información, creó los Estándares de Excelencia para el Uso Efectivo de la Información, que tienen por objetivo convertir a cada estudiante del sistema público de educación del país en un info-alfabeta; término utilizado por Lau (2006) para describir que todo estudiante debe poseer las habilidades para adquirir, analizar y utilizar la información de forma efectiva.

Los estándares presentados por el Programa de Servicios Bibliotecarios y de Información (2008) están organizados para trabajar el desarrollo de las destrezas de información en los diferentes currículos de las materias que se imparten en las escuelas públicas del país, mediante el establecimiento y evaluación de las metas y logros del estudiante. El Programa de Servicios Bibliotecarios y de Información también propone la aplicación de los valores éticos en el uso correcto de la información como parte del desarrollo de una sociedad democrática. El programa aspira a que los estudiantes, a través del dominio de las destrezas de información, desarrollen las habilidades necesarias para convertirse en usuarios de información efectiva.

En la guía curricular presentada en el 2007 por el Programa de Servicios Bibliotecarios y de Información, menciona la colaboración que debe existir entre los docentes y el maestro bibliotecario, ya que ambos comparten la responsabilidad de la enseñanza de las destrezas de información. Entre los deberes del maestro bibliotecario descritos en la guía se indica:

(...) trabajar con los maestros de manera colaborativa en la enseñanza de las destrezas así como en el diseño de sus cursos con el propósito de promover el diseño de tareas y trabajos de investigación que requieran de la búsqueda de información para completarse exitosamente. Además, propone la orientación de los maestros en cuanto a la forma efectiva de enseñar las destrezas de información. (GIDIC, 2007, p.4)

\section{bibliotecas}


Noraida Domínguez Flores

Eileene R. Adames Méndez
Bibliotecas. Vol 34, № 1, enero-junio, 2016, pp. 1-18. EISSN: 1659-3286

URL: http://www.revistas.una.ac.cr/index.php/bibliotecas/index

Lo anterior evidencia la necesidad de colaboración entre bibliotecarios y maestros, que es un tema que ha estado presente como elemento esencial en el proceso de enseñanza y aprendizaje. El logro de esta colaboración también ha sido analizado y documentado por varios profesionales de la información.

Entre la documentación revisada concerniente a la colaboración entre bibliotecarios y maestros, el denominador común encontrado radica en la importancia de que el bibliotecario tenga pleno conocimiento sobre las necesidades de información de la población a la que prestará sus servicios. En cuanto al tema de la colaboración, Husid (2013) explica cómo a través de la experiencia adquirida durante sus estudios graduados aprendió técnicas efectivas para lograr una colaboración productiva entre los bibliotecarios y el personal docente.

Husid (2013) recomienda al bibliotecario relacionarse con los currículos, estándares, mapas y unidades curriculares, ya que de esta forma conocerá las necesidades informativas de los docentes y podrá aportar materiales que enriquezcan el proceso de enseñanza. Por otro lado, en el 2013, Lambert, describe los pasos a seguir para lograr una colaboración efectiva con los maestros. Ella utilizó la oportunidad en la que las maestras le solicitaron libros para presentarles diferentes ideas de cómo realizar una integración curricular utilizando los recursos de la biblioteca.

Lambert creó unas actividades que los estudiantes se llevaron a sus casas, y a través de estas los estudiantes aprendieron cómo buscar libros de diversos géneros en la biblioteca. Los estudiantes accedían una página web que ella creó para completar una actividad y luego visitaban la biblioteca para buscar los libros y respondían a una prueba corta. Otra de las ideas consistió en utilizar el libro solicitado por la maestra para ofrecerles a los estudiantes una lección sobre la voz. Una última actividad desarrollada por Lambert fue la creación de una lección sobre puentes y creó una página web en la cual los estudiantes accedían y completaban ejercicios que también se llevaban a cabo en la sala de clases.

Mediante la creación de estas y otras actividades, Lambert logró la colaboración con diferentes maestros, permitiendo que la biblioteca se convirtiera en una extensión del salón de clases, alcanzando así que los docentes comprendieran la importancia y el valor añadido que tiene la integración de los recursos bibliotecarios al currículo y, por consecuente, al aprendizaje de sus estudiantes.

Trabajo similar presenta Kimmel (2012) quien discute sobre las experiencias encontradas a través de la interpretación de los datos analizados del proceso de colaboración entre ella, como bibliotecaria escolar y el equipo de maestros de segundo grado en una escuela elemental. El proceso de colaboración entre Kimmel y los maestros se extendió durante un año escolar. Esta bibliotecaria expone que durante las reuniones realizadas en el proceso de planificación con los maestros, se pudieron afrontar diversas situaciones de dificultad, como la tardanza de los maestros, falta de tiempo para planificar o la necesidad de realizar reuniones cortas (Kimmel, 2013).

La autora destaca el uso de reuniones mensuales con el personal docente para realizar la planificación y la integración de los recursos bibliotecarios. En el proceso de planificación, el rol del Kimmel fue esencial, ya que enriqueció las unidades de trabajo con diferentes recursos informativos. Al

\section{bibliotecas}


Noraida Domínguez Flores

Eileene R. Adames Méndez
Bibliotecas. Vol 34, № 1, enero-junio, 2016, pp. 1-18. EISSN: 1659-3286

URL: http://www.revistas.una.ac.cr/index.php/bibliotecas/index

finalizar, el análisis de los datos obtenidos en su estudio, Kimmel (2013) pudo identificar cinco pasos que se llevaron a cabo en las reuniones celebradas con los maestros.

El primer paso fue orienting (orientación), en este paso se presentaron los temas a trabajar, se organizaron las fechas de las próximas reuniones y se prepararon las agendas. El segundo paso Coordinating (coordinación), en el cual se ordenaron los horarios en los que los docentes utilizarían y compartirán los recursos. El making connections (haciendo conexiones) fue el paso en el que el bibliotecario presentó a los maestros todos los posibles materiales o actividades disponibles en la biblioteca y que, acorde con sus currículos, estos podían utilizar con los estudiantes. El cuarto paso fue descrito como making sense (haciendo sentido), se presentaron las metas académicas y se realizaron preguntas dirigidas a cómo alcanzarlas. Este paso permitió que cada maestro compartiera sus estrategias de enseñanza y avalúo. El último paso fue drifting (a la deriva). Este paso fue descrito por Kimmel (2013) como una actividad que permite que cada compañero compartiera información personal o de temas de su interés con los demás. Esto permitió que los participantes, nuevamente, analizaran los temas que dieron comienzo al proceso de planificación.

Como se evidencia en la literatura discutida, es significativo que el maestro bibliotecario sea percibido por los miembros de la comunidad escolar y la facultad como profesional de información y un enlace esencial para la adquisición de la información precisa y confiable. En este sentido, también se destaca la importancia del rol del maestro bibliotecario como colaborador en el proceso de planificación, diseño e implementación de diversas actividades, integradas al proceso de enseñanza y aprendizaje, con el fin de desarrollar las competencias de información de los estudiantes. Precisamente, la necesidad de que exista una percepción adecuada sobre el rol del maestro bibliotecario en los procesos de enseñanza y aprendizaje motivaron esta investigación.

\section{Objetivo, población y muestra}

Al considerar las diversas responsabilidades que tienen los profesionales en bibliotecología escolar, es necesario destacar que una muy importante es la enseñanza de las competencias de información. Para esto se debe utilizar como guía los Estándares de excelencia para el uso efectivo de la información publicados por el Departamento de Educación. En este documento se presentan cinco estándares que describen las expectativas específicas que se deben lograr en el estudiante y, según lo establecido en el documento, para el resultado de estos estándares y expectativas es necesaria la integración de las diversas actividades y experiencias en el currículo escolar.

No obstante, el poder cumplir con estas responsabilidades depende del personal de la biblioteca escolar y de los maestros, con sus conocimientos y apertura sobre el rol de los profesionales en bibliotecología escolar y de cómo pueden trabajar en equipo para el desarrollo de proyectos innovadores que le ofrezcan a los estudiantes el aprendizaje de las competencias que necesitan de por vida.

\section{bibliotecas}


Noraida Domínguez Flores

Eileene R. Adames Méndez
Bibliotecas. Vol 34, N 1, enero-junio, 2016, pp. 1-18. EISSN: 1659-3286

URL: http://www.revistas.una.ac.cr/index.php/bibliotecas/index

Para garantizar que los maestros cuenten con el conocimiento que necesitan sobre el rol de la biblioteca escolar, resulta necesario que, como parte de su preparación universitaria, se les ofrezcan experiencias curriculares que integren la colaboración con el personal de la biblioteca escolar. Por esto, el objetivo de la presente investigación es evaluar el impacto de un proyecto de colaboración entre estudiantes sub-graduados de la Facultad de Educación matriculados en el curso de PrePráctica y los estudiantes graduados de la Escuela de Ciencias y Tecnologías de la Información matriculados en el curso de Competencias Informacionales y Tecnológicas, en el conocimiento y percepción que tienen los estudiantes de la pre-práctica sobre el rol de la biblioteca escolar en el proceso de enseñanza y aprendizaje.

Un total de 19 estudiantes sub-graduados (17 mujeres y 2 hombres), entre las edades de 21 a 30 años, matriculados en el curso de pre-práctica de la Facultad de Educación participaron, junto a 17 estudiantes graduados matriculados en el curso de Competencias Informacionales y Tecnológicas de la EGCTI. Mediante la integración de un proyecto de colaboración en ambos cursos se buscaba responder a las siguientes preguntas de investigación:

$>\quad$ ¿Cuál es la percepción que tienen los estudiantes sub-graduados de la Facultad de Educación sobre el rol de profesionales en bibliotecología escolar en el proceso de enseñanza y aprendizaje?

¿Qué conocimiento tienen los estudiantes de pre-práctica sobre las competencias de información y cómo se integran en el currículo escolar?

¿Se educa a los estudiantes sub-graduados de la Facultad de Educación sobre el rol de los profesionales en bibliotecología escolar en el proceso de enseñanza y aprendizaje?

¿Cuál es el impacto de un proyecto de colaboración integrado al curso de Pre-Práctica de la Facultad de Educación y el curso de Competencias Informacionales y Tecnológicas de la Escuela Graduada de Ciencias y Tecnologías de la Información, en la percepción de los estudiantes pre-practicantes sobre el rol de los profesionales en bibliotecología escolar en el proceso de enseñanza y aprendizaje?

\section{Método y recopilación de datos}

Para responder a las preguntas de investigación se diseñó una metodología mixta (cualitativa y cuantitativa) que buscaba indagar sobre la percepción que tienen los estudiantes pre-practicantes, futuros maestros, sobre el rol de los profesionales en bibliotecología escolar.

\section{bibliotecas}


Noraida Domínguez Flores

Eileene R. Adames Méndez
Bibliotecas. Vol 34, № 1, enero-junio, 2016, pp. 1-18. EISSN: 1659-3286

URL: http://www.revistas.una.ac.cr/index.php/bibliotecas/index

Los datos fueron recopilados a través de los siguientes instrumentos:

- Un cuestionario administrado al inicio del semestre académico, el cual permitió identificar las percepciones iniciales de los estudiantes de pre-práctica sobre el rol de los profesionales en bibliotecología escolar. Un proyecto de colaboración fue integrado en un curso de Pre-Práctica de la Facultad de Educación. Como parte de este proyecto, los estudiantes sub-graduados y futuros maestros trabajaron junto a estudiantes graduados, futuros bibliotecarios escolares, matriculados en el curso de Competencias Informacionales y Tecnológicas que ofrece la Escuela Graduada de Ciencias y Tecnologías de la Información. A través de este proyecto, los estudiantes matriculados en ambos cursos tuvieron la oportunidad de colaborar en el diseño de una unidad curricular integrando los estándares del Programa de Bibliotecas Escolares del Departamento de Educación.

- Al finalizar el proyecto de colaboración, los estudiantes pre-practicantes completaron un cuestionario que permitió examinar el impacto del proyecto de colaboración en la percepción y conocimiento de los estudiantes participantes sobre el rol de los profesionales en bibliotecología escolar en el proceso de enseñanza y aprendizaje.

- Para la recopilación de datos cualitativos y revalidación de la información recopilada a través del cuestionario, los estudiantes participantes fueron invitados a participar de un grupo focal.

- Para identificar si los estudiantes de la Facultad de Educación son educados sobre el rol de la biblioteca escolar, se identificaron y seleccionaron al azar diversos prontuarios de cursos de concentración de la Facultad de Educación, para identificar si se incluye alguna experiencia que les permita a los estudiantes de esta facultad adquirir conocimientos sobre el rol de la biblioteca escolar en el proceso de enseñanza y aprendizaje.

A continuación se presentan y discuten los hallazgos de acuerdo con los instrumentos que fueron utilizados para la recopilación de datos. Más adelante se presentan las conclusiones, a la vez que se responden a las preguntas de investigación.

\section{Hallazgos}

\section{a. Cuestionario inicial}

Previo a iniciar el proyecto de colaboración que fue integrado en los cursos, los estudiantes matriculados en el curso de pre-práctica (futuros maestros) completaron un cuestionario, cuyos propósitos fueron:

$>\quad$ recopilar información socio-demográfica de los participantes;

identificar la experiencia que tuvieron con una biblioteca escolar durante sus años en escuela elemental, intermedia y superior;

$>\quad$ identificar su experiencia en una biblioteca a nivel universitario;

\section{bibliotecas}


Noraida Domínguez Flores

Eileene R. Adames Méndez
Bibliotecas. Vol 34, No 1, enero-junio, 2016, pp. 1-18. EISSN: 1659-3286

URL: http://www.revistas.una.ac.cr/index.php/bibliotecas/index

identificar si recibieron alguna enseñanza relacionada a la biblioteca escolar como parte de sus estudios universitarios;

> identificar la percepción que tienen sobre un profesional en bibliotecología escolar;

identificar si se visualizan trabajando en colaboración con un profesional en bibliotecología escolar, una vez que comiencen su experiencia laboral como maestros.

Como se mencionó anteriormente, el grupo de estudiantes sub-graduados consistió de 19 estudiantes, quienes provienen de 14 pueblos diferentes de la isla. En términos del tipo de escuela donde completaron sus estudios, la Tabla 1 presenta los resultados:

Tabla 1

Tipo de escuela donde cursaron estudios los participantes

\begin{tabular}{lcc}
\hline Nivel escolar & Pública & Privada \\
\hline Elemental $(\mathrm{k}-6 \mathrm{to})$ & 9 & 10 \\
Intermedia $(7 \mathrm{mo}-9 \mathrm{no})$ & 11 & 8 \\
Superior $(10 \mathrm{mo}-12 \mathrm{mo})$ & 10 & 9 \\
\hline
\end{tabular}

Fuente: elaboración propia de las autoras.

Según se evidencia, el número es casi equitativo en cuanto a los estudiantes que completaron sus estudios en una escuela pública y los que la completaron en una escuela privada. Sin embargo, para identificar la experiencia de los estudiantes con una biblioteca escolar resultaba necesario identificar si en las escuelas donde cursaron estudios tenían disponible una biblioteca (ver Tabla 2).

Tabla 2

Número de escuelas con Biblioteca Escolar

\begin{tabular}{lcc}
\hline Nivel escolar & Con Biblioteca Escolar & Sin Biblioteca Escolar \\
\hline Elemental $(\mathrm{k}-6 \mathrm{to})$ & 17 & 2 \\
Intermedia $(7 \mathrm{mo}-9 \mathrm{no})$ & 18 & 1 \\
Superior $(10 \mathrm{mo}-12 \mathrm{mo})$ & 18 & 1 \\
\hline
\end{tabular}

Fuente: elaboración propia de las autoras.

Considerando que casi el 100\% de los estudiantes cursaron estudios en escuelas que contaban con una biblioteca escolar, se esperaba que un alto porcentaje recordara haber tenido alguna experiencia visitando la biblioteca escolar o interactuando con su personal, ya sea dentro de la biblioteca o en el salón de clases. Para esto, se les cuestionó sobre qué tipo de actividades recordaba haber realizado en la biblioteca escolar. 
La Tabla 3 muestra el tipo de actividades que los participantes identificaron y el total de estudiantes que identificó cada una (encerrados en los paréntesis). Estas actividades fueron identificadas por cada nivel escolar (elemental, intermedio y superior), ya que se entiende que las actividades que se llevan a cabo en una biblioteca escolar pueden variar significativamente de acuerdo al nivel al cual pertenece.

Tabla 3

Actividades que los participantes llevaron a cabo en una biblioteca escolar

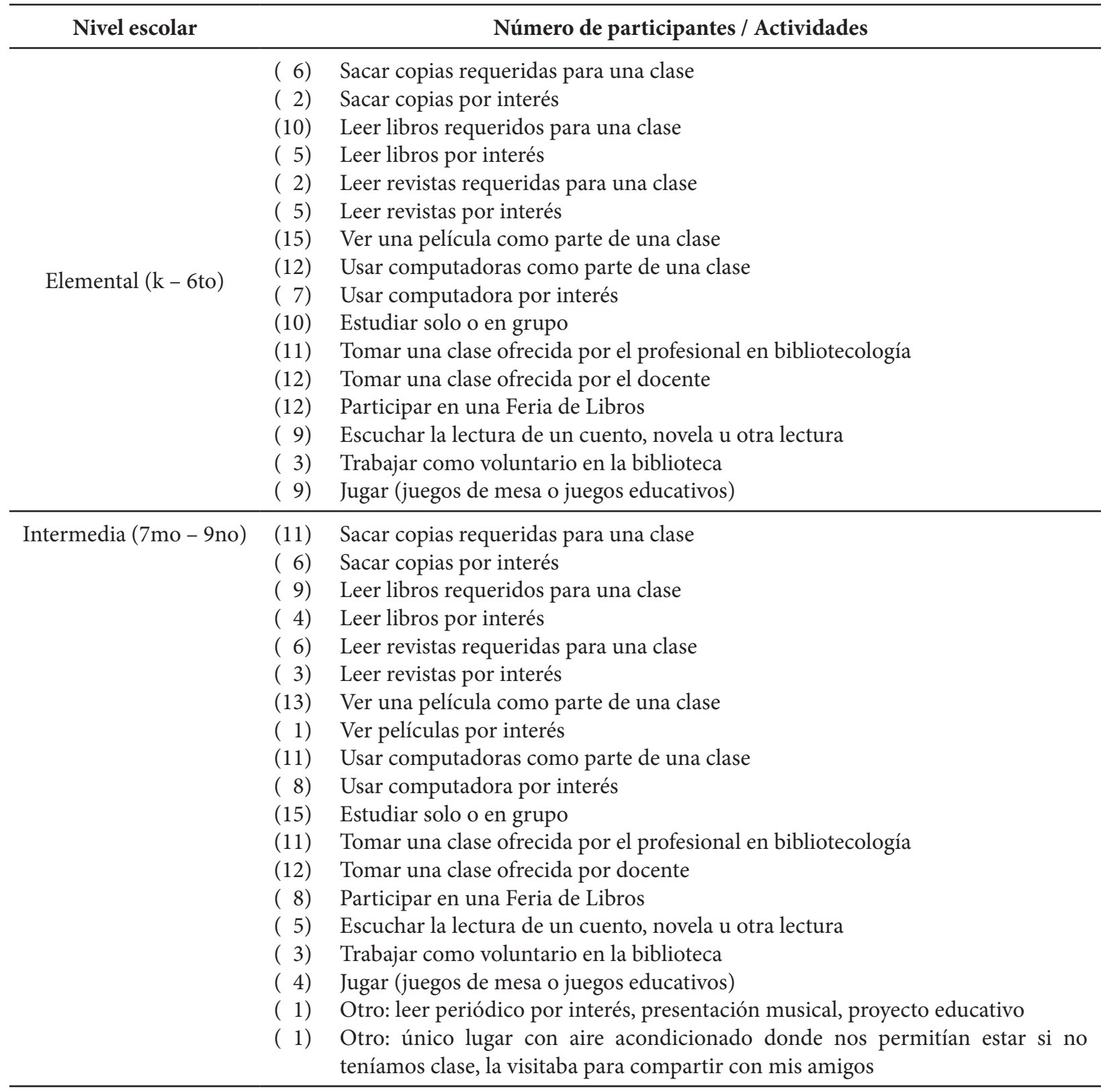


Noraida Domínguez Flores

Eileene R. Adames Méndez
Bibliotecas. Vol 34, No 1, enero-junio, 2016, pp. 1-18. EISSN: 1659-3286

URL: http://www.revistas.una.ac.cr/index.php/bibliotecas/index

(13) Sacar copias requeridas para una clase

( 7) Sacar copias por interés

(12) Leer libros requeridos para una clase

( 6) Leer libros por interés

( 7) Leer revistas requeridas para una clase

( 6) Leer revistas por interés

(10) Ver una película como parte de una clase

( 2) Ver películas por interés

Superior

$(10 \mathrm{mo}-12 \mathrm{mo})$

( 8) Usar computadoras como parte de una clase

( 8) Usar computadora por interés

(12) Estudiar solo o en grupo

(11) Tomar una clase ofrecida por el profesional en bibliotecología

(12) Tomar una clase ofrecida por el docente

( 7) Participar en una Feria de Libros

( 2) Escuchar la lectura de un cuento, novela u otra lectura

( 2) Trabajar como voluntario(a) en la biblioteca

( 1) Otro: conferencias, clubes

( 1) Otro: leer periódico por interés

( 1) Otro: Charlas sobre salud y drogas

Fuente: elaboración propia de las autoras.

Resulta necesario destacar que, según evidenciado en la Tabla 3, la experiencia de los participantes con la biblioteca escolar fue muy similar en cada uno de los niveles (elemental, intermedio y superior). En este sentido, se puede destacar que gran parte de los participantes tuvieron la experiencia de recibir clases u orientaciones ofrecidas por los profesionales en bibliotecología escolar, lo que evidencia que sí existía un nivel de colaboración entre los maestros de la sala de clases y los profesionales en bibliotecología escolar.

Ahora bien, se les preguntó sobre el impacto que tuvo en ellos la biblioteca en el proceso de enseñanza y aprendizaje. Solo el $47 \%$ de los estudiantes consideraban que la biblioteca escolar en los niveles K-6, tuvo algún impacto positivo en su proceso educativo, mientras que el 53\% no considera que tuvieran algún aprendizaje. Dentro de las razones por las cuales consideraron un impacto positivo mencionaron:

Tabla 4

Razones por las que la biblioteca escolar tuvo un impacto positivo

"la atmosfera de la biblioteca es distinta a la de un salón y permitía una manifestación académica más cómoda".

"fue un centro donde nos reunimos a estudiar después de las horas de clase".

"fueron muchas las horas que entre libros me divertí, esto marcó mi gusto por la lectura e inicié mis estudios universitarios en la literatura”.

"fue un recurso de mucha riqueza educativa y formadora para mis estudios".

"ayudó a utilizar herramientas para adquirir conocimientos nuevos".

Fuente: elaboración propia de las autoras. 
Noraida Domínguez Flores

Eileene R. Adames Méndez
Bibliotecas. Vol 34, No 1, enero-junio, 2016, pp. 1-18. EISSN: 1659-3286

URL: http://www.revistas.una.ac.cr/index.php/bibliotecas/index

Los participantes que consideraban que la biblioteca no tuvo un impacto positivo en ellos expresaron: "en mi opinión no tuvo debido a la falta de recursos y la manera o la actitud del bibliotecario", "no tuvo ningún impacto, creo yo, porque en ella [la bibliotecaria] no hizo nada que marcara mis años escolares", "no tuvo impacto, porque no le dieron énfasis para su verdadero uso, además que no nos dejaban entrar a ella porque podíamos romper algo o dañar".

En el nivel intermedio, solo el $37 \%$ mencionó que no tuvo un impacto positivo y un $63 \%$ indicó que sí. Algunas de las razones sobre porqué consideraban que tuvieron un aprendizaje fueron:

Tabla 5

Razones por las que la biblioteca escolar tuvo un impacto positivo

"tuve impacto positivo porque la atmósfera de la biblioteca es distinta a la de un salón y permitía una manifestación académica más cómoda".

"tuvo un impacto positivo en el aspecto social, ya que los estudiantes nos reuníamos para charlar o jugar juegos de mesa".

"en la biblioteca escolar existía buen trato hacia los estudiantes, era un buen ambiente, muchos recursos".

Fuente: elaboración propia de las autoras.

Las razones que expresaron los participantes de por qué no fue un impacto positivo para ellos son: "no tenían bibliotecarios o bibliotecas", "no las visitaban mucho", "no me impactó de forma positiva, era una persona hostil y aterradora". Mientras que en el nivel superior, el 63\% de los participantes contestó que sí tuvo un impacto efectivo y el $37 \%$ dijo que no. Entre las razones que expresaron de por qué fue efectivo, mencionaron:

Tabla 6

Razones por las que la biblioteca escolar tuvo un impacto positivo

"en la escuela superior los maestros involucraban mucho el uso de la biblioteca y pude aprovecharla al máximo tanto por requerimiento como por interés propio”.

"al entrar a la superior pude observar lo importante que es para nuestro desarrollo como estudiante".

Fuente: elaboración propia de las autoras.

Las razones por las que no consideran que tuvo un impacto positivo en su enseñanza y aprendizaje, en su mayoría, fueron: "porque no visitaban la biblioteca", "no se fomentaba el uso de la misma", un participante comentó que "no tuvo ningún impacto debido a que la biblioteca estaba escasa de recursos, computadoras dañadas, etc.".

\section{bibliotecas}


Noraida Domínguez Flores

Eileene R. Adames Méndez
Bibliotecas. Vol 34, No 1, enero-junio, 2016, pp. 1-18. EISSN: 1659-3286

URL: http://www.revistas.una.ac.cr/index.php/bibliotecas/index

Para contestar una de las preguntas iniciales, se les preguntó sobre la percepción que tenían de los bibliotecarios. La Tabla 7 resume las respuestas de los participantes:

Tabla 7

Percepción sobre bibliotecarios(as) escolares

\begin{tabular}{|c|c|c|}
\hline Nivel escolar & Aspectos positivos & Aspectos negativos \\
\hline $\begin{array}{c}\text { Elemental } \\
(\mathrm{k}-6 \text { to })\end{array}$ & $\begin{array}{l}\text { a) siempre a disposición de los estudiantes } \\
\text { b) persona muy buena, mujer dulce, pausada, } \\
\text { dispuesta a ayudar } \\
\text { c) "recuerdo que a mí me encantaba ir a la } \\
\text { biblioteca y compartir con la bibliotecaria, } \\
\text { era bien gentil y siempre dispuesta } \\
\text { ayudar" }\end{array}$ & $\begin{array}{l}\text { a) persona desorganizada } \\
\text { b) rígida y estricta } \\
\text { c) sin entusiasmo } \\
\text { d) no motivaba a leer ni estar en la biblioteca } \\
\text { e) llamaba mucho la atención } \\
\text { f) seria, no le gustaba interactuar con los niños }\end{array}$ \\
\hline $\begin{array}{c}\text { Intermedia } \\
(7 \mathrm{mo}-9 \mathrm{no})\end{array}$ & $\begin{array}{l}\text { a) atentas y serviciales } \\
\text { b) amables } \\
\text { c) facilitadora } \\
\text { d) dispuesta a ayudar } \\
\text { e) activa con la comunidad escolar }\end{array}$ & $\begin{array}{l}\text { a) desorganizadas } \\
\text { b) poco humanas y amables } \\
\text { c) no motivaban al estudiante }\end{array}$ \\
\hline $\begin{array}{c}\text { Superior } \\
(10 \mathrm{mo}-12 \mathrm{mo})\end{array}$ & $\begin{array}{l}\text { a) "buena bibliotecaria, responsable, aclara } \\
\text { dudas o preguntas" } \\
\text { b) "era una persona la cual estaba para } \\
\text { ayudar a los estudiantes a conseguir lo } \\
\text { que necesitaban para la escuela" } \\
\text { c) "recuerdo verla estudiar, planifi-car } \\
\text { la organización del espacio y siempre } \\
\text { conseguía libros de interés" }\end{array}$ & $\begin{array}{l}\text { a) rígida y desorganizada } \\
\text { b) amargada y peleona } \\
\text { c) "no permitía que en la hora de almuerzo } \\
\text { estuviera en la bibliote-ca, recuerdo. Sentía } \\
\text { que ella no quería compartir los libros y el } \\
\text { aire acondicionado conmigo", } \\
\text { d) regañaba si hacían ruidos excesi-vos, de lo } \\
\text { contrario no intervenía. }\end{array}$ \\
\hline
\end{tabular}

Fuente: elaboración propia de las autoras.

En otra pregunta, se les pidió a los participantes que definieran las Competencias de Información. El 20\% de los participantes mencionaron que desconocían lo que era y los restantes las definieron como distintos recursos o fuentes que existen para encontrar o adquirir información. Mencionaron, además, que "las competencias de información son las destrezas y el conocimiento que tenga uno sobre el manejo de informática y tecnología", "pienso que son las habilidades y los conocimientos que uno tiene sobre los recursos y tecnologías de información”. Por otro lado, el $74 \%$ de los participantes expresaron que nunca habían escuchado el concepto antes.

\section{bibliotecas}


Noraida Domínguez Flores

Eileene R. Adames Méndez
Bibliotecas. Vol 34, № 1, enero-junio, 2016, pp. 1-18. EISSN: 1659-3286

URL: http://www.revistas.una.ac.cr/index.php/bibliotecas/index

Siendo parte esencial del objetivo de la investigación el conocer si se ha discutido la inclusión de la biblioteca escolar como parte de los temas en algún curso del currículo académico de los participantes, se les preguntó si han incluido este tema en los cursos que han tomado como parte de su preparación académica. Solo el 32\% contestó que sí y un 68\% expresó que no.

Dentro de los temas relacionados a la biblioteca escolar ola actividad que se llevaron a cabo como discusión sobre el rol del profesional en bibliotecología escolar bibliotecario, los participantes mencionaron: la "participación de proyectos educativos en donde se orienta a los estudiantes, al igual que se comunican futuros maestros a través de páginas educativas", "en el curso de Principios de Investigación", "se mencionó una definición general de su rol en donde los niños podían buscar información". En este sentido, se evidenció que a los estudiantes de la Facultad de Educación no se les incluyen, como tema de alguno de sus cursos, una descripción del rol de la biblioteca escolar en el proceso de enseñanza y aprendizaje.

Considerando lo expresado por los participantes se percibe que solo mencionan la biblioteca escolar como el espacio donde los estudiantes pueden ir a localizar la información que necesiten o acceder recursos electrónicos que estén utilizando. No obstante, los participantes no expresaron haber participado en cursos donde se discutiera la biblioteca escolar como el espacio para la colaboración o el personal de la biblioteca escolar como un recurso clave

Por último, se indagó sobre las percepciones que tienen los participantes actualmente sobre el rol de los profesionales en bibliotecología escolar en el proceso de enseñanza y aprendizaje y expresaron: "es un recurso valioso para la educación, investigación y el conocimiento", "debe ser facilitador y motivador con estudiantes y maestros, "entiendo que el bibliotecario tiene una gran responsabilidad (junto al maestro) de fomentar el uso de la biblioteca y el encanto por el aprendizaje que se adquiere a través de un libro". Para finalizar el cuestionario, se les preguntó si como futuros maestros se visualizarían trabajando en equipo con el personal de la biblioteca escolar y un 95\% de los participantes contestaron que sí y solo un 5\% dijo que no.

\section{b. Cuestionario Final}

Los resultados de este cuestionario fueron comparados con los del primer cuestionario. En relación con la pregunta sobrela definición de las competencias de información, los participantes demostraron tener mayor comprensión que antes. Luego de culminado el proyecto de colaboración integrado en sus cursos, se presenta un aumento de conocimiento en un $26 \%$ del total de participantes.

En cuanto a la percepción que tienen actualmente sobre el rol del profesional en bibliotecología escolar en el proceso de enseñanza y aprendizaje, un 79\% de los participantes contestó que es un recurso importante para la integración y el mejor desarrollo de los estudiantes. Los porcientos restantes se dividen entre los que no contestaron nada (11\%) y los que no consideran efectivo esta integración (10\%). En cuanto a la interrogante sobre si consideraban que es importante integrar la enseñanza de la competencias de información en el currículo escolar, el 100\% de los participantes que tomaron el cuestionario respondió que sí.

\section{bibliotecas}


Noraida Domínguez Flores

Eileene R. Adames Méndez
Bibliotecas. Vol 34, № 1, enero-junio, 2016, pp. 1-18. EISSN: 1659-3286

URL: http://www.revistas.una.ac.cr/index.php/bibliotecas/index

Dentro de la pregunta relacionada con la percepción que tienen los estudiantes sobre el rol del profesional en bibliotecología escolar en el proceso de enseñanza y aprendizaje, un $79 \%$ de los participantes lo define como importante, necesario, facilitador de recursos y guía dentro del proceso. Un 10,5\% de los participantes no lo ve como un recurso y un 10,5\% no contestó nada. Al preguntarles si la percepción que tenían del rol bibliotecario cambió como resultado de la participación en este proyecto, un 68\% de los participantes contestó que sí y mencionaron: "sí, no sabía la importancia de este recurso", "sí, porque nunca había trabajado en colaboración con bibliotecarios y desconocía que eran las competencias de información", y "sí, antes pensaba que el maestro bibliotecario no tenía que intervenir en ningún aspecto en el salón de clase". Un 11\% de los participantes contestó que no había cambiado su percepción, un 16\% respondió que era la misma y un 5\% no respondió.

Al preguntarles si como futuros docentes, se visualizaban trabajando en equipo con el personal de la biblioteca escolar, el $21 \%$ de los estudiantes contestaron que no, mientras que el $79 \%$ de los participantes respondió que sí. Dentro de las respuestas que expresaron sobre por qué no se visualizaban trabajando como equipo (profesionales en bibliotecología escolar), solo dos participantes respondieron: "todavía no porque parecía como si la oportunidad fuera poca." El resto manifestó que es un recurso muy necesario e importante.

El 90\% de los participantes respondió que sí es necesario aprender sobre la biblioteca escolar como parte de sus estudios universitarios porque: "de esta manera se hará más fácil buscar y encontrar información para la clase", "esto contribuirá en gran medida al desarrollo profesional", "si voy a exponer a mis estudiantes a la misma tengo que conocerla yo primero". Un 5\% respondió que no y un $5 \%$ no estuvo presente en la administración del cuestionario.

\section{c. Grupo Focal}

El grupo focal estuvo compuesto por 10 estudiantes del total de los estudiantes participantes de bachillerato dela Facultad de Educación. Se comenzó pidiéndoles a los participantes que describieran en qué consistió el proyecto de colaboración en el cual participaron durante el semestre académico; un participante comentó

"nos presentaron de la unidad curricular en nuestro curso de la pre-práctica y después la profesora nos explicó que íbamos hacer ese proyecto colaborando con futuros bibliotecarios para ir adentrándonos en el sistema y que cuando seamos maestros vamos a tener que contar con los bibliotecarios y que es un buen recurso para nuestro salón sobre todo integrando la tecnología... y después entonces la otra fase era en el diseño de la unidad curricular que debíamos hacer planes en conjunto con nuestros bibliotecarios".

Expresaron que no sintieron una coordinación entre el grupo de los futuros maestros y los futuros bibliotecarios y que "iban a distintos pasos". Presentaron recomendaciones para próximos proyectos de esta índole como reunir a ambos grupos, explicar los criterios de los bibliotecarios y de los

\section{bibliotecas}


Noraida Domínguez Flores

Eileene R. Adames Méndez
Bibliotecas. Vol 34, № 1, enero-junio, 2016, pp. 1-18. EISSN: 1659-3286

URL: http://www.revistas.una.ac.cr/index.php/bibliotecas/index

maestros y establecer un contacto personal con los bibliotecarios. Durante el diálogo, mencionaron que utilizaron la aplicación de WhatsApp y el correo electrónico para ayudarles en la comunicación, pero aún así destacaron que no hubo una comunicación efectiva.

Durante este proceso, los futuros maestros mostraron gran confusión y desconocimiento sobre el rol del profesional en bibliotecología escolar y en múltiples ocasiones los participantes expresaron que no conocían su función dentro del proceso: "le enviamos el plan pero no sabíamos para qué ellos lo necesitaban". Por otra parte, mostraron gran interés en que se propicie una integración como esta colaboración entre futuros maestros y bibliotecarios en sus cursos universitarios.

Para todos ellos, esta fue la primera experiencia durante su desarrollo académico, en la cual han explorado la biblioteca y el profesional en bibliotecología escolar como aliados del desarrollo curricular. Dado a esta experiencia, consideraron que es necesario aprender sobre la biblioteca escolar como parte de sus estudios universitarios. Expresaron que como futuros docentes consideran la posibilidad de desarrollar este tipo de proyecto con la biblioteca escolar. Una de las participantes comentó que con este proyecto

"aprendimos que cuando estemos en una escuela debemos crear una comunicación y debemos crear como una relación de trabajo con el bibliotecario, porque a mí en ningún otro curso me habían enseñado ni me habían puesto a trabajar con bibliotecarios ni me habían enseñado la importancia de una biblioteca ni nada que en parte fue bueno".

En general, a través del grupo focal se pudo confirmar la información que fue recopilada a través del cuestionario administrado al finalizar el proyecto de investigación. Los participantes pudieron tener la experiencia de colaborar con futuros profesionales en bibliotecología escolar en la integración de las competencias de información en una unidad curricular. Dicha experiencia había sido la primera en toda su carrera universitaria, fue un proceso que al comienzo se hizo difícil de entender y también tuvieron que aprender a estar abiertos a colaborar con otros profesionales. En este sentido, la experiencia del grupo puso en evidencia lo que usualmente sucede en el ambiente escolar, en el cual el profesional en bibliotecología escolar busca desarrollar relaciones de colaboración con docentes, pero la falta de entendimiento sobre el rol de los primeros dificulta el inicio de este tipo de relación.

\section{Sílabos y Prontuarios}

Una de las fases de esta investigación fue evaluar los sílabos y prontuarios de diversos cursos que son parte del currículo académico del bachillerato en educación. Para la selección de los prontuarios a evaluar, se solicitaron las secuencias curriculares de todos los programas de bachillerato de la facultad de Educación. Una vez analizados todos los currículos ofrecidos, se lograron identificar siete prontuarios de cursos en que se podría discutir temas sobre el rol de los profesionales en bibliotecología escolar.

\section{bibliotecas}


Noraida Domínguez Flores

Eileene R. Adames Méndez
Bibliotecas. Vol 34, № 1, enero-junio, 2016, pp. 1-18. EISSN: 1659-3286

URL: http://www.revistas.una.ac.cr/index.php/bibliotecas/index

En cada prontuario se evaluaron sus objetivos, sus actividades, así como el bosquejo temático de cada uno. Se identificaron algunos temas relacionados a la alfabetización informacional, la tecnología y los recursos en línea, servicios y recursos bibliotecarios y recursos de información en línea y tecnología, pero todos estos temas estaban implícitos para ellos como estudiantes y no como futuros profesionales, ya que las experiencias ofrecidas no tenían como objetivo enseñar sobre la biblioteca escolar y su rol en los procesos de enseñanza. Por el contrario, los temas que fueron identificados eran parte de actividades que los estudiantes debían completar para cumplir con trabajos del curso que requerían el uso de recursos, servicios o tecnologías disponible en la biblioteca.

$\mathrm{Al}$ analizar cada prontuario, pudimos comprobar que en ninguno de estos cursos se incluye alguna experiencia que les permita a los estudiantes de esta facultad adquirir conocimientos sobre el rol de la biblioteca escolar en el proceso de enseñanza y aprendizaje. Por lo que se evidencia la necesidad de ofrecerles a estos estudiantes experiencias que le permitan aprender sobre las competencias de información y sobre el rol de los profesionales en bibliotecología escolar en la comunidad escolar.

\section{Conclusiones}

Luego de presentar y analizar los resultados obtenidos y al finalizar la integración de un proyecto de colaboración con los estudiantes practicantes (futuros maestros) y los estudiantes de bibliotecología, se presentan las conclusiones considerando cada una de las preguntas de investigación. En cuanto a la primera pregunta de investigación: ¿Cuál es la percepción que tienen los estudiantes subgraduados de la Facultad de Educación sobre el rol de los profesionales en bibliotecología escolar en el proceso de enseñanza y aprendizaje?, se puede concluir que la percepción principal de los estudiantes sub-graduados sobre el rol de estos profesionales es que son un recurso de apoyo. En este sentido, perciben que el personal de la biblioteca escolar está presente para facilitar recursos cuando los requieran. Es necesario destacar que los estudiantes de educación consideran la biblioteca escolar como un recurso valioso; no obstante, no entienden el rol de los profesionales en bibliotecología escolar como uno activo en los procesos de enseñanza y aprendizaje.

La segunda pregunta buscaba indagar sobre el conocimiento que tienen los estudiantes de prepráctica sobre las competencias de información y cómo se integran en el currículo escolar. En cuanto al tema de las competencias de información, se puede concluir que los estudiantes desconocen qué son las competencias de información, para qué son necesarias y cómo se deben desarrollar. Este hallazgo evidencia la necesidad de trabajar con los estudiantes (futuros maestros) las competencias de información que ellos deben desarrollar. El que los estudiantes sub-graduados posean competencias de información adecuadas, permitirá que reconozcan que como educadores, deben desarrollar las competencias de información que son tan necesarias hoy día y para el futuro.

Para responder a la tercera pregunta de investigación ¿Se educa a los estudiantes sub-graduados de la Facultad de Educación sobre el rol de los profesionales en bibliotecología escolar en el proceso de enseñanza y aprendizaje? Se evaluaron varios prontuarios de cursos del programa de

\section{bibliotecas}


Noraida Domínguez Flores

Eileene R. Adames Méndez
Bibliotecas. Vol 34, № 1, enero-junio, 2016, pp. 1-18. EISSN: 1659-3286

URL: http://www.revistas.una.ac.cr/index.php/bibliotecas/index

bachillerato de la Facultad de Educación. Considerando los resultados obtenidos, se puede concluir que los estudiantes sub-graduados de la Facultad de Educación no son educados sobre el rol de los profesionales en bibliotecología escolar en el proceso de enseñanza y aprendizaje. Esto evidencia la necesidad de revisar el currículo académico del bachillerato para crear y añadir cursos dirigidos a que los estudiantes de educación aprendan sobre el rol de la biblioteca escolar y de cómo pueden integrar a los profesionales de esta materia en los procesos de enseñanza y aprendizaje. De la misma forma, se debe educar sobre la importancia de integrar y colaborar con otros profesionales en la comunidad escolar, así como en las comunidades adyacentes. Este tipo de preparación podrá garantizar docentes con un entendimiento claro del rol de los profesionales que laboran en una comunidad escolar y de cómo pueden trabajar en colaboración para el beneficio de los estudiantes.

En cuanto a la última pregunta de investigación: ¿Cuál es el impacto de un proyecto de colaboración integrado al curso de Pre-Práctica de la Facultad de Educación y el curso de Competencias Informacionales y Tecnológicas de la Escuela Graduada de Ciencias y Tecnologías de la Información, en la percepción de los estudiantes pre-practicantes sobre el rol de los profesionales en bibliotecología escolar en el proceso de enseñanza y aprendizaje? Se puede concluir que, a pesar de los contratiempos y la falta de comunicación efectiva que algunos estudiantes expresaron haber experimentado, el proyecto tuvo un impacto positivo en la percepción de los futuros docentes sobre el rol de los profesionales en bibliotecología escolar en los procesos de enseñanza y aprendizaje.

La experiencia de poder colaborar con futuros profesionales en bibliotecología escolar permitió que los estudiantes de educación obtuvieran un mejor conocimiento sobre la labor que se realiza en una biblioteca escolar y las responsabilidades que comparten con profesionales en bibliotecología en torno al proceso educativo, especialmente sobre las competencias de información. En este sentido, el mayor impacto del proyecto de colaboración es que el grupo de estudiantes, una vez se inicien en sus carreras magisteriales, podrán iniciar sus propios proyectos de colaboración con el personal de la biblioteca escolar, permitiendo así ofrecer nuevas experiencias de aprendizaje.

Finalmente, se espera que este tipo de proyecto continúe desarrollándose y que la Facultad de Educación considere integrar en su currículo académico un curso enfocado a educar a sus estudiantes sobre el trabajo en equipo, el desarrollo de relaciones de colaboración con otros profesionales de la comunidad escolar y sobre el rol de la biblioteca escolar en los procesos de enseñanza y aprendizaje. Con esta preparación, se garantiza que los maestros del futuro enriquezcan sus currículos con nuevas experiencias de aprendizaje para sus estudiantes.

\section{Nota de Agradecimiento}

Se agradece el apoyo y colaboración de la Dra. Lizzette Velázquez Rivera, profesora de la Facultad de Educación de la Universidad de Puerto Rico, Recinto de Río Piedras, quien incorporó el proyecto en su curso e hizo posible que se llevara a cabo esta investigación.

\section{bibliotecas}


Noraida Domínguez Flores

Eileene R. Adames Méndez
Bibliotecas. Vol 34, No 1, enero-junio, 2016, pp. 1-18. EISSN: 1659-3286

URL: http://www.revistas.una.ac.cr/index.php/bibliotecas/index

\section{Referencias}

Departamento de Educación de Puerto Rico. (2008). Estándares de contenido y expectativas de grado: Programa de Servicios Bibliotecarios. [Artículo en línea] Recuperado el 4 de mayo de 2015 de http://www.de.gobierno.pr/ofrecimiento-academico/241-servicios-bibliotecarios/2021estandares-de-excelencia-para-el-uso-efectivo-de-la-informacion

Departamento de Educación de Puerto Rico. (2012). Dí para el uso del Internet y recursos de tecnología del Departamento de Educación de Puerto Rico. [Artículo en línea] Recuperado el 4 de mayo de 2015 de http://www.de.gobierno.pr/sites/de.gobierno.pr/files/Manual\%20de\%20Uso\%20 de\%20Internet\%202012.pdf

Díaz, F., y Hernández, G. (2002). Constructivismo y el aprendizaje significativo. México: Mc Graw Hill.

EDUTEKA. (2006). Estándares en TIC para docentes. [Artículo en línea] Recuperado el 4 de mayo de 2015 de http://www.eduteka.org/EstandaresNETSDocentes2000 1.php

Husid, W. (2013). Collaboration: Make it happen in your school. Library Media Connection, 31(4), 42-44.

Lambert, N. (2013). Connecting with the curriculum: Library collaborations that work for students and teachers. Library Media Connection, 32(3), 48-49.

Lau, J. (2006). Directrices para el desarrollo de habilidades informacionales: propuesta IFLA Abreviada 1. Revista brasilera de Biblioteconomia e Documentacco, 2 (1), 83-104

Kimmel, S. (2012). Collaboration as school reform: Are there patterns in the chaos of planning with teachers? School Library Research, 151-15.

Kimmel, S. (2013). Pass the chocolate planning with teachers. Knowledge Quest, 42(1), 48-51.

Smith III, M., Schiano, A., y Lattanzio, E. (2014). Beyond the classroom. Knowledge Quest, 42(3), 20-29.

\section{Notas sobre las autoras}

\footnotetext{
* PhD Noraida Domínguez Flores. Escuela Graduada de Ciencias y Tecnologías de la Información de la Universidad de Puerto Rico. Correo electrónico: noraida.dominguez@upr.edu

** MIS Eileene R. Adames Méndez. Escuela Graduada de Ciencias y Tecnologías de la Información de la Universidad de Puerto Rico. Correo electrónico: eileene.adames@gmail.com

*** MIS Damaris Arocho Molina. Escuela Graduada de Ciencias y Tecnologías de Información de la Universidad de Puerto Rico. Correo electrónico: damarisarochomolina@gmail.com
}

\section{bibliotecas}

